\title{
Combined Ang-2 and VEGF Targeting Therapies in Renal Cell Carcinoma*
}

\author{
Nikolett Molnar ${ }^{1}$, Dietmar W. Siemann ${ }^{1,2}$ \\ ${ }^{1}$ Department of Pharmacology and Therapeutics, University of Florida College of Medicine, Gainesville, USA; ${ }^{2}$ Department of Ra- \\ diation Oncology, University of Florida College of Medicine, Gainesville, USA. \\ Email: nmolnar@ufl.edu
}

Received July 23 ${ }^{\text {rd }}, 2013$; revised August 22 ${ }^{\text {nd }}, 2013$; accepted August $30^{\text {th }}, 2013$

Copyright (C) 2013 Nikolett Molnar, Dietmar W. Siemann. This is an open access article distributed under the Creative Commons Attribution License, which permits unrestricted use, distribution, and reproduction in any medium, provided the original work is properly cited.

\begin{abstract}
Angiogenesis, the growth of new vessels from pre-existing ones, is an important feature of tumor growth that has been exploited as a therapeutic target in oncology. Given its key role in facilitating blood vessel sprouting, VEGF has been a major focus of anti-angiogenic strategies, but the observation of resistance in some clinical trials utilizing such agents has led to a search for new or complementary targets in angiogenesis process. The Angiopoietin/Tie2 pathway and in particular the Angiopoietin-2 (Ang-2) ligand which is critically involved in the destabilization of normal vasculature, has been identified as one such target. The current study investigated the potential benefits of combining an Ang-2 targeted therapy with small molecule VEGF targeted agents (Sunitinib, Cediranib) in a human renal cell carcinoma model. The results showed that while both Ang-2 and VEGF interference on their own impaired tumor growth and new blood vessel formation, the combination of agents that targeted both pathways resulted in significantly superior anti-tumor and anti-angiogenic effects.
\end{abstract}

Keywords: Angiopoietin-2; Angiogenesis; Anti-Angiogenic Agent; Combination Therapy; Vascular Endothelial Growth Factor

\section{Introduction}

Angiogenesis, the formation of new blood vessels from pre-existing ones, is an important process in normal vascular development and physiological conditions such as wound healing, reproduction and the menstrual cycle [1]. The importance of angiogenesis not only in physiological but in pathological conditions has been well established [2]. A growing tumor cannot sustain its growth without the initiation and continued maintenance of active angiogenesis $[3,4]$. The resulting vasculature is distinct from normal vasculature both functionally and structurally and such differences allow for the targeting of tumor vasculature with limited effects on normal vasculature $[5,6]$.

Numerous clinical trials that seek to impair the induction of new blood vessels in tumors are ongoing $[7,8]$.

\footnotetext{
"Financial Support: These investigations were supported in part by a grant from the National Cancer Institute (Public Health Service Grant CA089655) and National Institutes of Health (T32 Training Grant 5T32 CA009126-33)

Conflict of Interest: No conflict of interest.
}

Many agents targeting Vascular Endothelial Growth Factor (VEGF) are now commonly used in the clinic; particularly in diseases such as kidney cancer $[9,10]$. However, resistance to such therapies may occur [11-13], likely due to the redundancy of signaling pathways involved in the activation of sprouting angiogenesis [14]. The search for new or complementary targets in the angiogenic process to circumvent resistance and such therapies is therefore being actively pursued $[7,11,12]$.

The Angiopoietin/Tie2 pathway has been shown to be important both in physiological and pathological angiogenesis including tumor angiogenesis [15]. Briefly, Ang-1 and Ang-2 are secreted proteins that interact with the Tie2 receptor either in a paracrine (Ang-1) or autocrine (Ang-2) manner; Ang-1 is expressed and secreted by peri-endothelial mural cells (smooth muscle cells, pericytes) while Ang-2 is expressed and secreted by endothelial cells [16]. Both angiopoietins bind the Tie2 receptor with similar affinities at the same site of the IgG-like and EGF-like domains [17,18]. These ligands, however, have opposing functions. Ang1-Tie2 signaling 
controls vessel quiescence, while Ang2-Tie2 association allows for vessel plasticity [19]. Elevated Ang-2 levels have been associated with advanced disease, progression and poor prognosis in several cancers including renal cell carcinoma [20-22]. Ang-2 serum levels significantly increase in patients compared to healthy individuals, and patients with more advanced disease show significantly higher levels of Ang-2 compared to patients with earlier stage disease [23-27].

Angiogenesis can be considered to be a two-step process: 1 ) the normal vasculature is destabilized by loosening the endothelial and peri-endothelial cell contacts in the vasculature (Angiopoietin/Tie2 pathway) at which point 2) pro-angiogenic factors such as VEGF activate the endothelium to proliferate and form new vessels [28]. Currently the majority of FDA approved anti-angiogenic agents target the second step of this process. VEGF signaling interference has been investigated in particular but inhibition of other angiogenesis associated signaling pathways is also being pursued [11]. In general, antiangiogenic agents have been found to be complementary to conventional cancer therapies, however, there are some patients who do not respond or stop responding after prolonged treatment with such agents [11-13]. In lieu of this observation and given the role of the Angiopoietin/Tie2 axis in angiogenesis, there has been growing interest in anti-angiogenic treatment approaches that selectively target both Ang-2 and VEGF [15,29-33].

The current study evaluated the combination of an investigational anti-Ang-2 monoclonal antibody with two small molecule tyrosine kinase inhibitors against the VEGF pathway, Sunitinib and Cediranib. The former is a multikinase inhibitor that is FDA approved for kidney cancer [34] while the latter is a VEGFR specific small molecule inhibitor that is currently in clinical development [35]. The effects of utilizing these agents alone or in combination on tumor development and angiogenesis initiation were evaluated in an aggressive human renal cell carcinoma model.

\section{Materials and Methods}

\subsection{Reagents}

MECA-32 was purchased from BioLegend (San Diego, CA), AlexaFluor 594 was purchased from Invitrogen (Grand Island, NY). VectaShield mounting medium with DAPI was purchased from Vector Labs Inc. (Burlingame, CA). Tissue-Tek OCT Compound was purchased from Sakura Finetek (Torrance, CA). 2-methylbutane was obtained from Thermo Fisher Scientific (Waltham, MA).

\subsection{Cell Culture}

The human clear cell renal cell carcinoma cell line
Caki-2 was received as a gift from Dr. Susan Knox (Stanford University). Caki-2 cells were grown in Dulbecco's modified minimum essential medium (DMEM, Invitrogen) supplemented with $10 \%$ fetal bovine serum (FBS, Invitrogen), 1\% penicillin-streptomycin (Invitrogen), and $1 \%$ 200-mmol/L L-glutamine (Invitrogen). Cells were maintained at $37^{\circ} \mathrm{C}$ in a $5 \% \mathrm{CO} 2$-incubator. The cells were used between passages 2 and 10 .

\subsection{Drug Preparation}

Anti-Angiopoietin-2 monoclonal antibody was kindly provided by MedImmune, LLC. Stock solutions $(5 \mathrm{mg}$ / $\mathrm{ml}$ ) of the antibody were diluted to working concentrations in sodium citrate buffer solution. Stock solutions were kept at $-80^{\circ} \mathrm{C}$ and working concentrations at $4^{\circ} \mathrm{C}$. Cediranib (AstraZeneca, Wilmington, DE) was stored at $4^{\circ} \mathrm{C}$ and prepared fresh daily in $10 \%$ volume Tween 80 and $1 \mathrm{M}$ HEPES. Sunitinib (LC Laboratories, Woburn, MA) was stored at $-20^{\circ} \mathrm{C}$ and prepared fresh daily in stock and diluent buffers of citric acid monohydrate and sodium citrate dihydrate at $\mathrm{pH} 6.8$ and 3.2 respectively at 1:7 stock to diluent solution ( $\sim \mathrm{pH} 3.3)$ and acidified to $\mathrm{pH}$ 1.0. Sunitinib was dissolved, and the solution adjusted to $\mathrm{pH} 3.5$.

\subsection{Intradermal Assay}

All in vivo procedures were conducted in agreement with a protocol approved by the University of Florida Institutional Animal Care and Use Committee. Female athymic nu/nu mice were injected intradermally with $10^{5}$ Caki-2 cells (10 $\mu \mathrm{l}$ volume) at four sites on the ventral surface. Beginning the day prior to tumor cell injection, mice were treated with 1) daily oral gavage of either Sunitinib $(10 \mathrm{mg} / \mathrm{kg})$ or Cediranib $(2 \mathrm{mg} / \mathrm{kg}), 2)$ IP injection of anti-Ang-2 antibody ( $2 \mathrm{mg} / \mathrm{kg}$ ) every 3 days or 3) the combination of either Sunitinib or Cediranib plus the anti-Ang-2 antibody. At the end of the treatment (six days post tumor cell inoculation) the mice were then euthanized. Tumors were measured using calipers and tumor volumes $\left(\mathrm{mm}^{3}\right)$ were calculated using the formula:

tumor volume $=\pi / 6 \times$ diameter $_{1} \times$ diameter $_{2} \times$ height

Skin flaps were then removed and vessels growing into tumor nodules were counted using a Leica MZ16F dissecting microscope with Leica KL 1500 LCD fiber optic illuminator (Leica Microsystems Inc., Buffalo Grove, IL) at $2.5 \times$ original magnification (1-3). Images were captured with a Retiga EXi Fast1394 digital CCD camera (QImaging, British Columbia, Canada) and OpenLab5 software (PerkinElmer Inc., Waltham, MA). Statistical significance between control and treated groups was determined using the Mann-Whitney U-Test at $\mathrm{p}<0.05$. 


\subsection{Immunohistochemistry}

Intradermal tumors were freshly frozen in OCT and methylbutane and sectioned at $5 \mu \mathrm{m}$ thickness using a Leica CM 3050S cryostat (Leica Microsystems Inc., Buffalo Grove, IL). Sections were placed on superfrost plus gold slides (Thermo Fisher Scientific Inc., Waltham, MA) and kept at $-80^{\circ} \mathrm{C}$ until immunohistochemical staining. Tissue sections were fixed with acetone for $10 \mathrm{~min}$, blocked in $2 \%$ normal horse serum in $1 \times \mathrm{TBS}$, and incubated overnight at $4^{\circ} \mathrm{C}$ with MECA-32. Secondary antibody AlexaFluor 594 was added onto slides for $1 \mathrm{hr}$. Tissue sections were imaged with a Zeiss Axioplan 2 imaging microscope (Carl Zeiss, Inc., Thornwood, NY) with EXFO X-Cite 120 light source (Lumen Dynamics Group Inc., Ontario, Canada). Images were taken with a Retiga EXi Fast digital CCD camera (Qimaging, British Columbia, Canada) and processed using OpenLab5 software (PerkinElmer Inc., Waltham, MA); Rhodamine for MECA-32/AlexaFluor594 and DAPI filters were used. Up to ten random fields were examined on each tumor section and the number of vessels in each field was counted using a $20 \times$ objective. Statistical significance between control and treated groups was determined using the Mann-Whitney U-Test at $\mathrm{p}<0.05$.

\section{Results}

\subsection{Treatments Targeting Both Ang-2 and VEGF Result in Significantly Greater Impairment of Tumor Growth than Either Therapy Alone}

Figure 1(a) shows that treatment with either the antiAng-2 antibody or the VEGFR1-3 inhibitor Cediranib resulted in significant reductions in tumor volumes compared to those of untreated mice, 3.5- and 3.8-fold ( $\mathrm{p}<$ $0.05)$ respectively. However, the combination of these two therapies resulted in an even greater impairment of tumor growth; at the end of the treatment period tumors treated with the anti-Ang-2 antibody plus Cediranib were 54.6-fold $(\mathrm{p}<0.0001)$ smaller than untreated control tumors. Furthermore, the tumors of mice with the combination of agents were also significantly smaller than those of mice treated solely with the anti-Ang-2 antibody (15.6-fold, $\mathrm{p}<0.0001$ ) or Cediranib (14-fold, $\mathrm{p}<0.01$ ). Similar results were seen when the anti-Ang-2 antibody and the multikinase inhibitor Sunitinib were combined (Figure 1(b)). Both agents administered individually impaired tumor growth; tumor volumes assessed at the end of the treatment period were found to be 1.5-fold ( $\mathrm{p}=$ 0.06 ) 4.6-fold ( $\mathrm{p}<0.0001$ ) smaller that untreated control tumors for anti-Ang-2 antibody and Sunitinib treatments respectively. However, the combination of the two agents once again led to a greater reduction in tumor volume (15.8 fold, $\mathrm{p}<0.0001$ ) compared to control tumors as

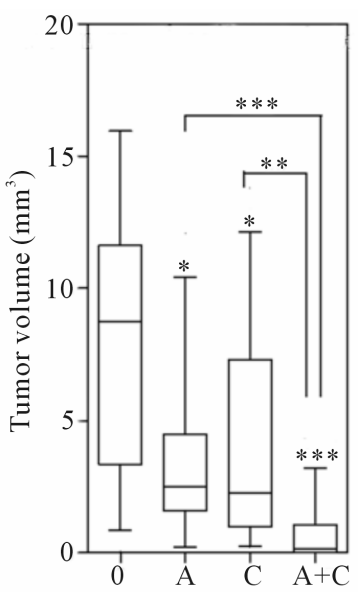

(a)

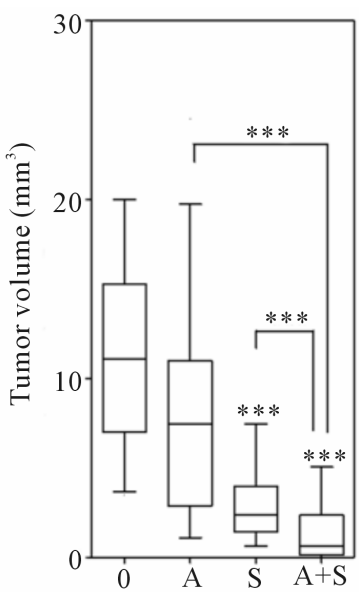

(b)
Figure 1. Effect of Ang-2 and VEGF treatment on tumor growth. Mice were injected intradermally with Caki-2 renal cell carcinoma cells and beginning the day prior to tumor cell injection, were treated with (a) Cediranib (2 mg/kg), (b) Sunitinib (10 mg/kg), ((a) and (b)) anti-Ang-2 antibody (2 $\mathrm{mg} / \mathrm{kg}$ ), or the combination of either (a) Cediranib plus the anti-Ang-2 antibody or (b) Sunitinib plus the anti-Ang-2 antibody. Tumor volumes were assessed 6 days after tumor cell inoculation. Line, median; bar 10 - 90 percentile; Control (0) $(\mathrm{n}=20)$, anti-Ang-2 antibody $(\mathrm{A})(\mathrm{n}=16)$, Cediranib $(C)(n=16)$, Sunitinib $(n=16)$, anti-Ang-2 antibody + Cediranib $(A+C)(n=16)$, anti-Ang-2 antibody + Sunitinib $(\mathrm{A}+\mathrm{S})(\mathrm{n}=\mathbf{2 0}) .{ }^{*} \mathrm{p}<0.05 ;{ }^{* *} \mathrm{p}<0.01 ;{ }^{* * *} \mathrm{p}<0.0001$; Mann-Whitney U-Test.

well as tumors in mice treated with only the anti-Ang- 2 antibody (10.7-fold, $\mathrm{p}<0.0001)$ or Sunitinib (3.4-fold, $\mathrm{p}<$ $0.0001)$.

\subsection{Combined Ang-2 and VEGF Targeting Significantly Impairs Tumor Cell Induced Angiogenesis}

To evaluate the effect of Ang-2 and VEGF directed therapies administered alone or in combination on Caki-2 renal cell carcinoma cell induced angiogenesis an intradermal angiogenesis assay was used (Figure 2). The results showed that while the anti-Ang-2 antibody and Cediranib treatments individually reduced the number of vessels growing into the tumor nodules by $1.6-(\mathrm{p}<0.01)$ and 1.5 -fold $(\mathrm{p}<0.01)$ respectively, the combination treatment led to an even greater reduction in blood vessel number (2.7-fold ( $\mathrm{p}<0.0001)$ compared to control) (Figure 2(a)). Similar results were seen when the antiAng-2 antibody was combined with Sunitinib (Figure 2(b)). While the anti-Ang-2 antibody and Sunitinib each decreased the number of vessels induced by Caki-2 tumor cells (1.7- and 1.8-fold ( $\mathrm{p}<0.0001)$ respectively), combining the therapies led to a significantly greater reduction in the number of blood vessels induced than was achieved with either agent alone (3.5-fold $(\mathrm{p}<$ 
0.0001), compared to control tumors). Furthermore, in both studies (Figures 2(a) and (b)), the combination treat- ment was significantly more effective at reducing the formation of tumor cell induced blood vessels than either the anti-Ang-2 antibody or the VEGF directed therapy when used alone.

The impact of VEGF and Ang-2 directed therapy on the vasculature within Caki-2 tumors was evaluated using immunohistochemistry (Figure 3). The results showed that treatment with the anti-Ang-2 antibody or Cediranib reduced the number of blood vessels within the tumor nodules 2.5 - and 1.4 -fold $(\mathrm{p}<0.01)$ respectively and the combination treatment led to a 3.5 -fold $(\mathrm{p}<0.01)$ reduction in intratumor blood vessels (Figure 3(a)). A similar analysis performed on the tumors of mice treated with the anti-Ang-2 antibody alone or in combination with Sunitinib (Figure 3(b)) showed that the combination reduced the number of blood vessels detected in the tumor nodules 3.7-fold $(\mathrm{p}<0.05)$ compared to the $2-(\mathrm{p}<$ $0.05)$ and 1.7 -fold $(\mathrm{p}=0.057)$ decrease in tumor blood
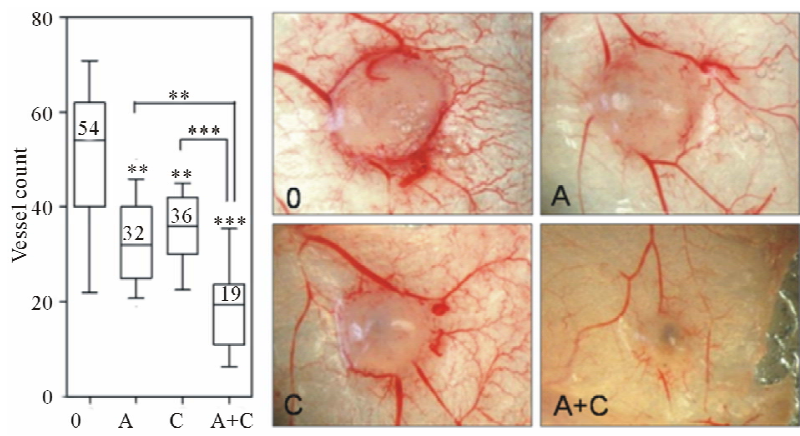

(a)
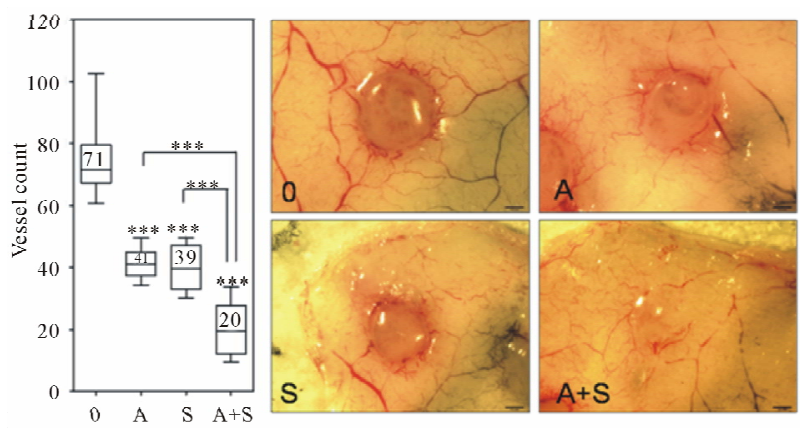

(b)

Figure 2. Effect of Ang-2 and VEGF treatments on blood vessels induction by Caki-2 tumor cells. Mice were treated as described in Figure 1 and the number of peripheral vessels growing into the tumor nodules was counted 6 days after tumor cell inoculation. Line, median; bar 10-90 percentile; Control (0) $(n=16)$, anti-Ang-2 antibody (A) $(n=$ 16), Cediranib (C) $(n=16)$, Sunitinib $(n=12)$, anti-Ang-2 antibody + Cediranib $(A+C)(n=16)$, anti-Ang-2 antibody + Sunitinib $(A+S)(n=16) ;{ }^{* *} p<0.01 ; * * * 0<001$; MannWhitney U-Test. Representative images were taken at $2.5 \times$ magnification.
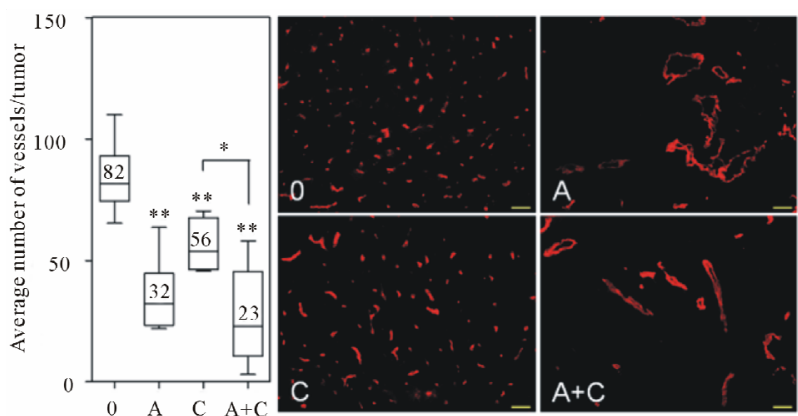

(a)
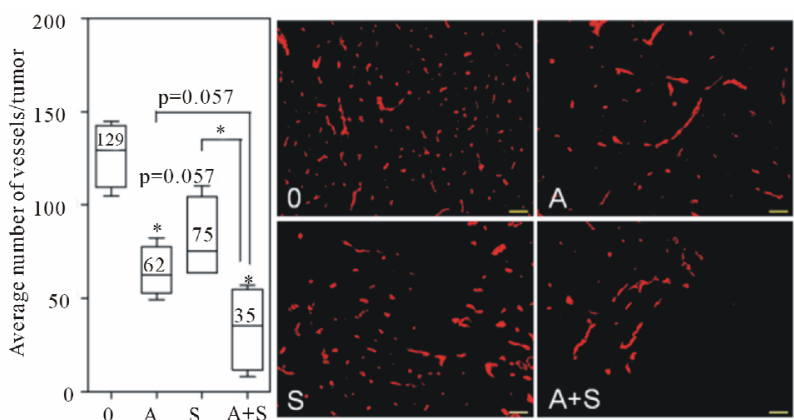

(b)

Figure 3. Effect of Ang-2 and VEGF treatments on tumor core blood vessels assessed by immunohistochemistry. Mice were treated as described in Figure 1. Six days post tumor cell inoculation frozen tumor sections were stained with MECA-32 and up to 10 random fields/tumor were evaluated. Line, median number of vessels; bar 10 - 90 percentile; Control (0) $(n=4$ - 6), anti-Ang-2 antibody (A) $(n=4$ - 6), Cediranib $(C)(n=6)$, Sunitinib $(n=4)$, anti-Ang-2 antibody + Cediranib $(A+C)(n=6)$, anti-Ang-2 antibody + Sunitinib $(A+S)(n=4)$ "p $<0.05 ;{ }^{* *} p<0.01$; Mann-Whitney $\mathrm{U}$-Test. Representative images of the median of each group are shown. Images taken with a Zeiss Axioplan Imaging2 microscope with a $20 \times$ objective; scale bar $=140 \mu \mathrm{m}$.

vessels noted in the tumors of mice treated with the anti-Ang-2 antibody or Sunitinib alone.

\section{Discussion}

Angiogenesis is an important feature of tumor growth that has been considered to be a potential target for cancer therapy for decades. Angiogenesis can be separated into two main events: 1) the destabilization of normal vasculature, or the loosening of endothelial and periendothelial cell contacts, and 2) the activation of the endothelium to proliferate and form new vessels. The Angiopoietin/Tie2 axis is responsible for the first step in angiogenesis or the vessel destabilization while pro-angiogenic factors such as VEGF activate the endothelial cells. Currently FDA approved anti-angiogenic agents to target the VEGF pathway and has been shown to complement conventional therapies such as chemotherapy [36], however the issues of lack of patient response and tumor rebound due to acquired resistance have raised 
concerns in the clinic [11-13].

Another strategy to interfere with tumor angiogenesis is to target the Angiopoietin/Tie2 pathway [15]. Ang-2 is abundantly present in many tumors and its expression appears to correlate with poor disease prognosis [22]. It is also conceivable that Ang-2 and VEGF targeting may be complementary and that Ang-2/Tie2 targeting may circumvent patient resistance to VEGF targeting therapy. While endothelial cell activation to form sprouts occurs in response to a variety of pro-angiogenic factors in addition to VEGF, the initial destabilization of vasculature through the Ang-2/Tie2 axis is currently known as a nonredundant pathway. In the current study the effects of inhibiting these two pathways through the combination of VEGF targeted agents and an anti-Ang-2 monoclonal antibody were evaluated in an aggressive and highly vascularized, VHL mutated, human renal cell carcinoma model. Two different VEGF targeted agents were evaluated, the small molecule multi tyrosine kinase inhibitor Sunitinib that is FDA approved for treatment of metastatic kidney cancer and the VEGFR specific small mol cule inhibitor Cediranib that is in clinical development for a variety of solid tumors.

The results of the present study support the notion that the efficacies of Ang-2 and VEGF targeted therapies may be complementary. When either Cediranib or Sunitinib was combined with the anti-Ang-2 antibody, the combination treatment showed superior anti-tumor and antiangiogenic effects compared to any of the agents used on their own (Figures 1 and 2). The enhanced treatment efficacy was likely not only a consequence of a reduction in the ability of the renal cells to induce the initiation of vessel growth toward the tumor mass (Figure 2), but also a consequence of an impairment of vascular development within the tumors themselves (Figure 3). Taking these findings together lends additional support to therapeutic intervention strategies seeking to maximize antitumor efficacy through combination treatments directed at the multiple components comprising tumor angiogenesis.

\section{Acknowledgements}

The authors thank MedImmune, LLC for kindly providing the anti-Ang-2 monoclonal antibody and Marda Jorgensen (University of Florida Tissue Core) for assistance with the immunohistochemistry studies.

\section{REFERENCES}

[1] M. Papetti and I. M. Herman, "Mechanisms of Normal and Tumor-Derived Angiogenesis," American Journal of Physiology Cell Physiology, Vol. 282, No. 5, 2002, pp. C947-C970. http://dx.doi.org/10.1152/ajpcell.00389.2001

[2] J. Folkman, "Angiogenesis: An Organizing Principle for Drug Discovery?” Nature Reviews Drug Discovery, Vol.
6, No. 4, 2007, pp. 273-286. http://dx.doi.org/10.1038/nrd 2115

[3] J. Folkman, "Tumor Angiogenesis: Therapeutic Implications," New England Journal of Medicine, Vol. 285, No. 21, 1971, pp. 1182-1186. http://dx.doi.org/10.1056/NEJM197111182852108

[4] E. Goldmann, "The Growth of Malignant Disease in Man and the Lower Animals, with Special Reference to the Vascular System," Proceeding of the Royal Society of Medicine, Vol. 1, Surgery Section, 1908, pp. 1-13.

[5] P. Vaupel, "Abnormal Microvasculature and Defective Microcirculatory Function in Solid Tumors," In: D. W. Siemann, Ed., Vascular Targeted Therapies in Oncology, Wiley Ltd., West Sussex, 2006, pp. 9-29. http://dx.doi.org/10.1002/0470035439.ch2

[6] J. Denekamp, "Vascular Endothelium as the Vulnerable Element in Tumours," Acta Radiological Oncology, Vol. 23, No. 4, 1984, pp. 217-225. http://dx.doi.org/10.3109/02841868409136015

[7] K. M. Cook and W. D. Figg, "Angiogenesis Inhibitors: Current Strategies and Future Prospects," CA: A Cancer Journal for Clinicians, Vol. 60, No. 4, 2010, pp. 222-243. http://dx.doi.org/10.3322/caac.20075

[8] ClinicalTrials.gov, "Anti-Angiogenic Therapy: Cancer," U.S. National Institutes of Health, 2013. http://clinicaltrials.gov

[9] C. Coppin, C. Kollmannsberger, L. Le, F. Porzsolt and T. J. Wilt, "Targeted Therapy for Advanced Renal Cell Cancer (RCC): A Cochrane Systematic Review of Published Randomized Trials," British Journal of Urology International, Vol. 108, No. 10, 2011, pp. 1556-1563.

[10] S. Bellou, G. Pemtheroudakis, C. Murphy and T. Fotsis, "Anti-Angiogenesis in Cancer Therapy: Hercules and Hydra," Cancer Letters, Vol. 338, No. 2, 2013, pp. 219-228. http://dx.doi.org/10.1016/j.canlet.2013.05.015

[11] S. Loges, T. Schmidt and P. Carmeliet, "Mechanisms of Resistance to Anti-Angiogenic Therapy and Development of Third-Generation Anti-Angiogenic Drug Candidates," Genes and Cancer, Vol. 1, No. 1, 2010, pp. 12-25. http://dx.doi.org/10.1177/1947601909356574

[12] G. Bergers and D. Hanahan, "Modes of Resistance to Anti-Angiogenic Therapy," Nature Reviews Cancer, Vol. 8, No. 8, 2008, pp. 592-603. http://dx.doi.org/10.1038/nrc2442

[13] J. M. Ebos, C. R. Lee and R. S. Kerbel, "Tumor and Host-Mediated Pathways of Resistance and Disease Progression in Response to Antiangiogenic Therapy," Clinical Cancer Research, Vol. 15, No. 16, 2009, pp. 50205025. http://dx.doi.org/10.1158/1078-0432.CCR-09-0095

[14] N. Ferrara, "Pathways Mediating VEGF-Independent Tumor Angiogenesis," Cytokines \& Growth Factor Reviews, Vol. 21, No. 1, 2010, pp. 21-26.

http://dx.doi.org/10.1016/j.cytogfr.2009.11.003

[15] D. Gerald, S. Chintharlapalli, H. G. Augustin and L. E. Benjamin, "Angiopoietin-2: An Attractive Target for Improved Antiangiogenic Tumor Therapy," Cancer Research, Vol. 73, No. 6, 2013. pp. 1649-1657. http://dx.doi.org/10.1158/0008-5472.CAN-12-4697 
[16] P. C. Maisonpierre, et al., "Angiopoietin-2, a Natural Antagonist for Tie2 that Disrupts in Vivo Angiogenesis," Science, Vol. 277, No. 5322, 1997, pp. 55-60. http://dx.doi.org/10.1126/science.277.5322.55

[17] U. Fiedler, et al., "Angiopoietin-1 and Angiopoietin-2 Share the Same Binding Domains in the Tie-2 Receptor Involving the First Ig-Like Loop and the Epidermal Growth Factor-Like Repeats," The Journal of Biological Chemistry, Vol. 278, No. 3, 2003, pp. 1721-1727. http://dx.doi.org/10.1074/jbc.M208550200

[18] W. A. Barton, D. Tzvetkova and D. B. Nikolov, "Structure of the Angiopoietin-2 Receptor Binding Domain and Identification of Surfaces Involved in Tie2 Recognition," Structure, Vol. 13, No. 5, 2005, pp. 825-832. http://dx.doi.org/10.1016/j.str.2005.03.009

[19] P. Saharinen and K. Alitalo, "The Yin, The Yang, and the Angiopoietin-1," The Journal of Clinical Investigation, Vol. 121, No. 6, 2011, pp. 2157-2159. http://dx.doi.org/10.1172/JCI58196

[20] M. Yamakawa, et al., "Expression of Angiopoietins in Renal Epithelial and Clear Cell Carcinoma Cells: Regulation by Hypoxia and Participation in Angiogenesis," American Journal of Physiology Renal Physiology, Vol. 287, No. 4, 2004, pp. F649-F657. http://dx.doi.org/10.1152/ajprenal.00028.2004

[21] M. J. Currie, et al., "Expression of the Angiopoietins and Their Receptor Tie2 in Human Renal Clear Cell Carcinomas; Regulation by the Von Hippel-Lindau Gene and Hypoxia," Journal of Pathology, Vol. 198, No. 4, 2002, pp. 502-510. http://dx.doi.org/10.1002/path.1228

[22] C. R. Tait and P. F. Jones, "Angiopoietins in Tumours: The Angiogenic Switch,” Journal of Pathology, Vol. 204, No. 1, 2004, pp. 1-10. http://dx.doi.org/10.1002/path.1618

[23] M. Sie, et al., "The Angiopoietin 1/Angiopoietin 2 Balance as a Prognostic Marker in Primary Glioblastoma Multiforme," Journal of Neurosurgery, Vol. 110, No. 1, 2009, pp. 147-155. http://dx.doi.org/10.3171/2008.6.17612

[24] A. J. Lind, et al., "Angiopoietin 2 Expression is Related to Histological Grade, Vascular Density, Metastases, and Outcome in Prostate Cancer," Prostate, Vol. 62, No. 4, 2005, pp. 394-399. http://dx.doi.org/10.1002/pros.20163

[25] I. Helfrich, et al., "Angiopoietin-2 Levels Are Associated with Disease Progression in Metastatic Malignant Melanoma," Clinical Cancer Research, Vol. 15, No. 4, 2009, pp. 1384-1392. http://dx.doi.org/10.1158/1078-0432.CCR-08-1615

[26] K. M. Detjen, et al., "Angiopoietin-2 Promotes Disease Progression of Neuroendocrine Tumors," Clinical Cancer
Research, Vol. 16, No. 2, 2010, pp. 420-429. http://dx.doi.org/10.1158/1078-0432.CCR-09-1924

[27] J. H. Park, et al., "Serum Angiopoietin-2 as a Clinical Marker for Lung Cancer," CHEST, Vol. 132, No. 1, 2007, pp. 200-206. http://dx.doi.org/10.1378/chest.06-2915

[28] G. Bergers and L. E. Benjamin, "Tumorigenesis and the Angiogenic Switch," Nature Reviews Cancer, Vol. 3, No. 6, 2003, pp. 401-410. http://dx.doi.org/10.1038/nrc1093

[29] H. Hashizume, et al., "Complementary Actions of Inhibitors of Angiopoietin-2 and VEGF on Tumor Angiogenesis and Growth," Cancer Research, Vol. 70, No. 6, 2010, pp. 2213-2223.

http://dx.doi.org/10.1158/0008-5472.CAN-09-1977

[30] R. Dandu, et al., "Design and Synthesis of Dihydroindazolo[5,4-A]Pyrrolo[3,4-C]Carbazole Oximes as Potent Dual Inhibitors of TIE-2 and VEGF-R2 Receptor Tyrosine Kinases," Bioorganic \& Medicinal Chemistry Letters, Vol. 18, No. 6, 2008, pp. 1916-1921. http://dx.doi.org/10.1016/j.bmcl.2008.02.001

[31] Y. J. Koh, et al., "Double Antiangiogenic Protein, DAAP, Targeting VEGF-A and Angiopoietins in Tumor Angiogenesis, Metastasis, and Vascular Leakage," Cancer Cell, Vol. 18, No. 2, 2010, pp. 171-184. http://dx.doi.org/10.1016/j.ccr.2010.07.001

[32] J. L. Brown, et al., "A Human Monoclonal Anti-ANG2 Antibody Leads to Broad Antitumor Activity in Combination with VEGF Inhibitors and Chemotherapy Agents in Preclinical Models," Molecular Cancer Therapeutics, Vol. 9, No. 1, 2010, pp. 145-156. http://dx.doi.org/10.1158/1535-7163.MCT-09-0554

[33] C. C. Leow, et al., "MEDI3617, a Human Anti-Angiopoietin 2 Monoclonal Antibody, Inhibits Angiogenesis and Tumor Growth in Human Tumor Xenograft Models," International Journal of Oncology, Vol. 40, No. 5, 2012, pp. 1321-1330.

[34] V. L. Goodman, et al., "Approval Summary: Sunitinib for the Treatment of Imatinib Refractory or Intolerant Gastrointestinal Stromal Tumors and Advanced Renal Cell Carcinoma," Clinical Cancer Research, Vol. 13, No. 5, 2007, pp. 1367-1373. http://dx.doi.org/10.1158/1078-0432.CCR-06-2328

[35] A. X. Zhu, et al., "Efficacy, Safety, Pharmacokinetics, and Biomarkers of Cediranib Monotherapy in Advanced Hepatocellular Carcinoma: A Phase II Study," Clinical Cancer Research, Vol. 19, No. 6, 2013, pp. 1557-1566. http://dx.doi.org/10.1158/1078-0432.CCR-12-3041

[36] R. S. Kerbel, “Antiangiogenic Therapy: A Universal Chemosensitization Strategy for Cancer?" Science, Vol. 312, No. 5777, 2006, pp. 1171-1175. http://dx.doi.org/10.1126/science. 1125950 